\title{
Bak instead of Bax plays a key role in metformin-induced apoptosis $s$ in HCT116 cells
}

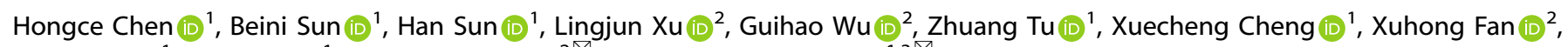 \\ Zihao Mai $\mathbb{D}^{1}$, Qiling Tang ${ }^{1}$, Xiaoping Wang $\mathbb{i D}^{2 \bowtie}$ and Tongsheng Chen $\mathbb{D}^{1,3^{凶}}$
}

(c) The Author(s) 2021

Metformin (Met) exhibits anticancer ability in various cancer cell lines. This report aims to explore the exact molecular mechanism of Met-induced apoptosis in HCT116 cells, a human colorectal cancer cell line. Met-induced reactive oxygen species (ROS) increase and ROS-dependent cell death accompanied by plasma membrane blistering, mitochondrial swelling, loss of mitochondrial membrane potential, and release of cytochrome c. Western blotting analysis showed that Met upregulated Bak expression but downregulated Bax expression. Most importantly, silencing Bak instead of Bax inhibited Met-induced loss of mitochondrial membrane potential, indicating the key role of Bak in Met-induced apoptosis. Live-cell fluorescence resonance energy transfer (FRET) analysis showed that Met unlocked the binding of Mcl-1 to Bak, and enhanced the binding of Bim to Bak and subsequent Bak homo-oligomerization. Western blotting analysis showed that Met enhanced AMPK phosphorylation and Bim expression, and compound C, an inhibitor of AMPK, inhibited Met-induced Bim upregulation. Although Met increased the expression of Bcl-xL, overexpression of $\mathrm{Bcl}-\mathrm{xL}$ did not prevent Met-induced apoptosis. In summary, our data demonstrate for the first time that Met promotes ROS-dependent apoptosis by regulating the Mcl-1-Bim-Bak axis.

Cell Death Discovery (2021)7:363; https://doi.org/10.1038/s41420-021-00755-y

\section{INTRODUCTION}

Metformin (Met), a biguanide derivative derived from herbal medicine and being used as the first-line oral drug for the treatment of type 2 diabetes, has been demonstrated to prevent a variety of tumors [1]. Numerous preclinical, epidemiological and clinical studies have shown that Met is associated with the reduction of cancer incidence and cancer-related death [1, 2]. Met is thought to have two potential anti-tumor pathways: AMPKdependent and AMPK-independent pathways. In the AMPKdependent pathway, Met directly inhibits mitochondrial respiratory chain complex I to limit oxidative phosphorylation, thereby reducing the amount of ATP production, and increasing AMP/ATP ratio and AMPK phosphorylation and activation. AMPK activation inhibits mTOR signaling via phosphorylating and activating p53, leading to autophagy and apoptosis $[3,4]$. In the AMPKindependent pathway, Met reduces insulin and insulin-like growth factor-1 $[5,6]$. However, the exact mechanism by which Met reduces tumor growth is still unclear.

Colorectal cancer (CRC) ranks third in terms of incidence and the second most common cause of cancer death in the world, characterized by poor prognosis and low 5-year survival rate of $11 \%[7,8]$. CRC patients are typically treated with conventional cytotoxic chemotherapy, targeted therapy and anti-PD-1 immunotherapy $[9,10]$. However, most CRC patients are either inherently insensitive to therapeutic treatment or easy to acquire resistance upon relapse. There is a critical need to develop novel and more effective CRC therapies [11]. Epidemiological studies have found that Met can reduce the incidence, mortality and recurrence risk of CRC in patients with diabetes, thus increasing the survival benefit and the effect of chemotherapy drugs $[12,13]$. Experimental studies have shown that Met inhibits proliferation of CRC cells and suppresses intestinal polyp growth in ApcMin ${ }^{\prime+}$ mice, suggesting that Met may be a novel candidate for CRC [14].

The $\mathrm{BCl}-2$ family proteins play a key role in regulating apoptosis. Bax and Bak are key effectors to initiate apoptotic pathway. Many kinds of tumors exhibit overexpression of anti-apoptotic proteins ( $\mathrm{MCl}-1, \mathrm{BCl}-\mathrm{xL}$, and $\mathrm{BCl}-2)$, near all anti-tumor drugs targeting the $\mathrm{Bcl}-2$ family have focused on the inhibition of $\mathrm{Bcl}-\mathrm{xL}$ and $\mathrm{BCl}-2$ [15]. It is reported that Bid most efficiently activates Bak than Bim, whereas Bim exhibits stronger affinity for Bax than Bid [16]. The balance between proapoptotic members and anti-apoptotic components maintains normal physiological activities of cells $[17,18]$. Met induces apoptosis by downregulating $\mathrm{Bcl}-2$ and upregulating Bax in ovarian cancer [19,20], and induces cell death by inhibiting $\mathrm{Mcl}-1$ in hypoglycemia [21]. Further studies are needed to elucidate the precise mechanisms by which Met induces cell death.

This report aims to explore how Met regulate $\mathrm{BCl}-2$ family proteins to induce apoptosis in HCT116 cells, a kind of CRC cells. Our data firmly proved for the first time that the Mcl-1-Bim-Bak axis mediated the Met-induced apoptosis. Met activates AMPK to increase Bim expression, in turn activates Bak, leading to the

\footnotetext{
${ }^{1}$ MOE Key Laboratory of Laser Life Science \& Guangdong Provincial Key Laboratory of Laser Life Science, College of Biophotonics, South China Normal University, 510631 Guangzhou, China. ${ }^{2}$ Department of Pain Management, The First Affiliated Hospital of Jinan University, 510632 Guangzhou, China. ${ }^{3}$ SCNU Qingyuan Institute of Science and

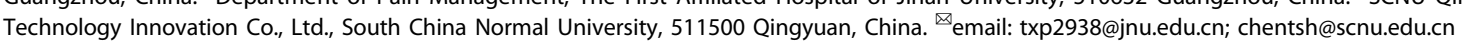

Received: 10 September 2021 Revised: 25 October 2021 Accepted: 3 November 2021

Published online: 22 November 2021 
release of cytochrome $c$ and subsequent activation of downstream apoptosis signaling pathway. Interestingly, $\mathrm{Bcl}-\mathrm{xL}$ is not involved in Met-induced apoptosis though Met-increased $\mathrm{BCl}-\mathrm{xL}$ expression.

\section{RESULTS}

Met induces plasma membrane blistering and cytotoxicity

CCK-8 assay showed that treatment with different concentrations (10-100 mM) of Met for $24 \mathrm{~h}$ induced dose-dependent cytotoxicity (Fig. 1A). To verify whether Met induces apoptosis and necrosis, we imaged the cells stained by Hoechst/PI, and the STS-treated cells were used as the positive control (Figs. 1B, S1B). Morphological observation showed that Met-treated cells contracted, and more than $50 \%$ of the cells showed vesicles over the whole cells surface, but kept their membrane integrity (PI negative), and also exhibited obvious nuclear chromatin pyknosis and karyolysis. After small vesicles become bullae, PI entered the cells (Fig. S1A). The vesicles on the cell membrane surface may be apoptotic bodies. Taken together, Met has potent in vitro anticancer capability against HCT116 cells.

\section{Met induces ROS-dependent apoptosis in HCT116 cells}

In order to further verify whether Met-induced apoptosis, the percentage of apoptotic cells was quantitatively analyzed by using flow cytometry for the cells stained by Annexin-V/PI. The percentage of apoptotic cells includes early apoptotic cells and late apoptotic cells or necrotic cells. Compared with the control group, a dose-dependent increase of the apoptotic cells was observed after $24 \mathrm{~h}$ incubation with different concentrations of Met (Fig. 2A). The caspase activity assay by using Western blotting and enzyme-labeling instrument detection showed that Metinduced activation of caspase-3, caspase- 8 and caspase-9 (Figs. 2B, S2), which was further verified by Western blotting analysis on the Met-induced PARP cleavage (Fig. 2C), an important indicator of cell apoptosis and caspase-3 activation [22]. Z-VAD (a broadspectrum caspase inhibitor) inhibited Met-induced cell death (Fig. 2D), further demonstrating that Met-induced casapses- dependent intrinsic apoptosis. Met or STS induced a significantly increased intracellular reactive oxygen species (ROS) (Fig. 2E), and NAC (reactive oxygen species inhibitor) significantly inhibited Metinduced cell death (Fig. 2F), indicating that Met-induced ROS production and ROS-dependent apoptosis.

Bak instead of Bax plays a key role in Met-induced apoptosis We used JC-1 probe to detect whether Met-induced loss of mitochondrial membrane potential (Fig. 3A). Fluorescence microscope imaging revealed that Met-induced loss of mitochondrial membrane potential in concentration-dependent fashion. Microscopic imaging also showed that Met caused mitochondrial swelling (Fig. 3B), further indicating that Met impaired mitochondrial function. Bax and Bak, as the main effectors of Bcl-2 family proteins, play a key role in the intrinsic apoptosis pathway [23]. Western blotting analysis showed that Met upregulated Bak expression but downregulated Bax expression in HCT116 cells (Fig. 3C). Moreover, silencing Bak instead of Bax inhibited Metinduced loss of mitochondrial membrane potential (Fig. 3D), indicating that Bak instead of Bax plays a key role in Met-induced apoptosis. We next assessed the distribution of cytochrome $\mathrm{c}$ in single living cells expressing GFP-cytochrome $c$ by using fluorescence microscopic imaging (Fig. 3E). In control cells, cytochrome c mainly concentrated in mitochondria. In Mettreated cells, cytochrome c was released from mitochondria and distributed uniformly in the cytoplasm.

Next, quantitative fluorescence resonance energy transfer (FRET) measurements were performed in living cells coexpressing CFP-Bak and YFP-Bak to explore whether Metinduced Bak homo-oligomerization. Figure 3F shows the fluorescence images of representative cells co-expressing CFP-Bak and YFP-Bak in the absence (control) or presence of Met, and the corresponding pixel-to-pixel pseudo-color $E_{D}$ and $R c$ images as well as their histograms. The two $E_{D}-R_{C}$ binding curves from at least 108 cells were saturable when $R c$ was larger than 4 , and the 0.765 of $E_{D \max }$ in Met-treated cells was larger than the 0.609 of $E_{D \max }$ in control cells (Fig. $3 \mathrm{~F}$ ), indicating that Met-increased Bak homo-oligomerization.
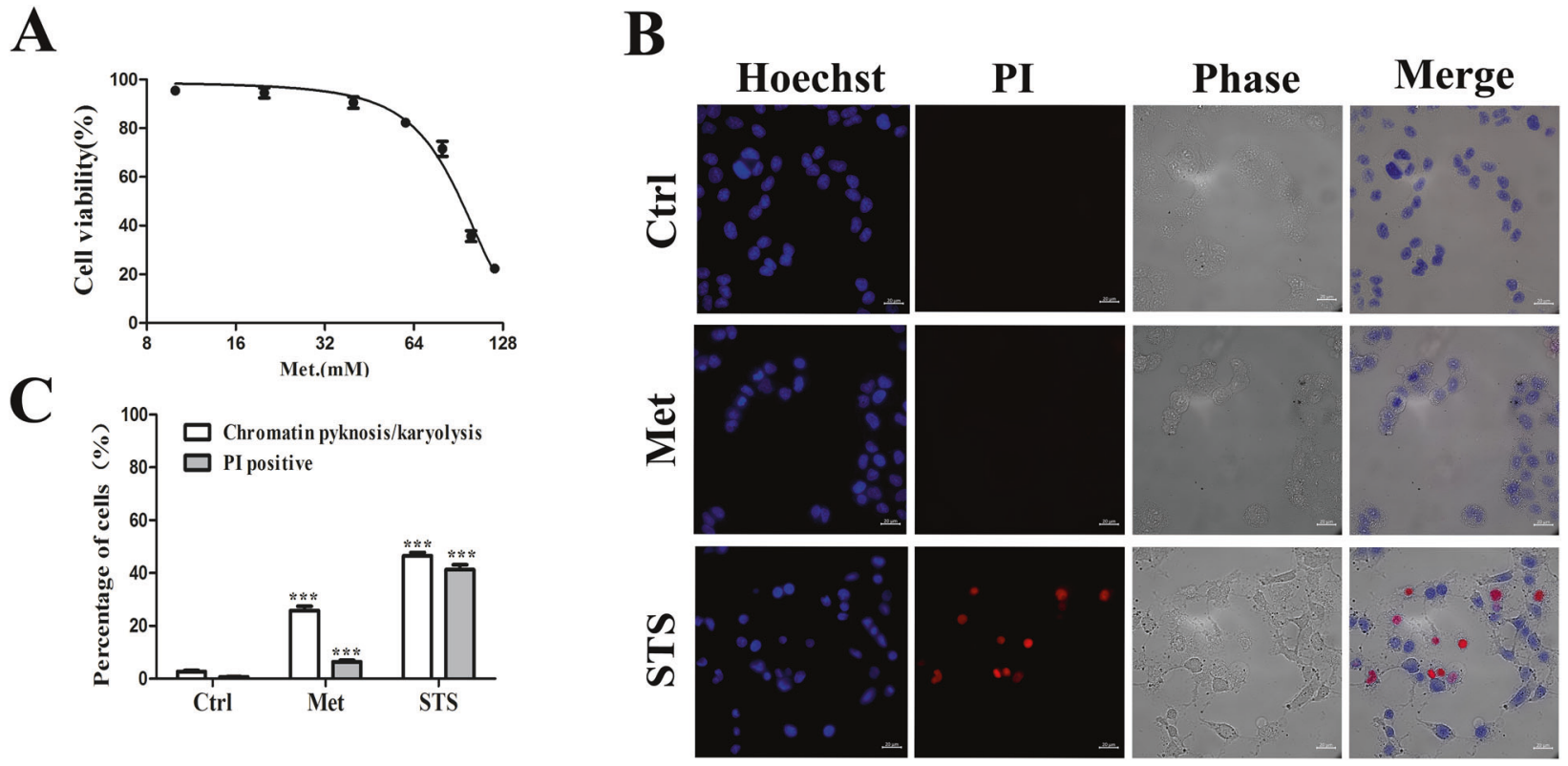

Fig. 1 Met induces plasma membrane blistering and cytotoxicity. A Met-induced dose-dependent cytotoxicity measured by CCK-8 assay. Cells were seeded into 96-well microplate and incubated with different concentration of Met (0-100 mM) for $24 \mathrm{~h}$. B Representative image of cells stained with Hoechst/PI staining after treatment with $60 \mathrm{mM}$ Met for $24 \mathrm{~h}$, or $1 \mu \mathrm{M}$ STS for $6 \mathrm{~h}$. C Statistical percentages of cells with nuclear chromatin pyknosis/karyolysis and PI positive respectively from at least 500 cells. All data are expressed with the mean \pm SEM of three independent experiments. ${ }^{* *} p<0.001$ compared with the control group. 
A

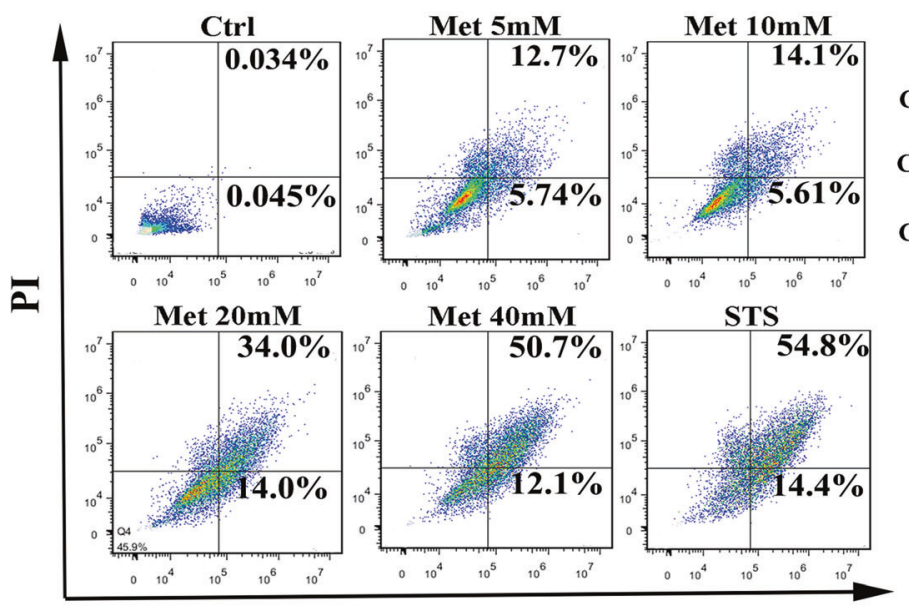

B

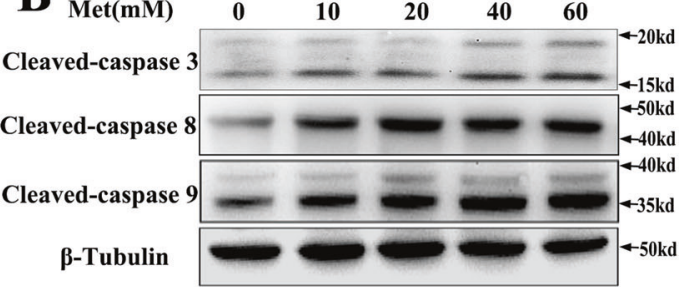

C

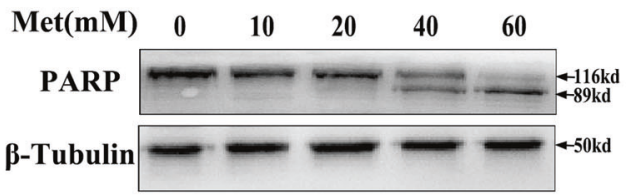

AnnexinV-FITC

D

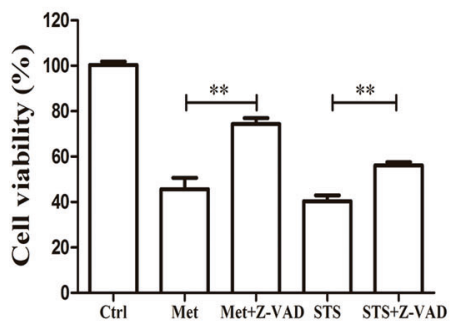

E

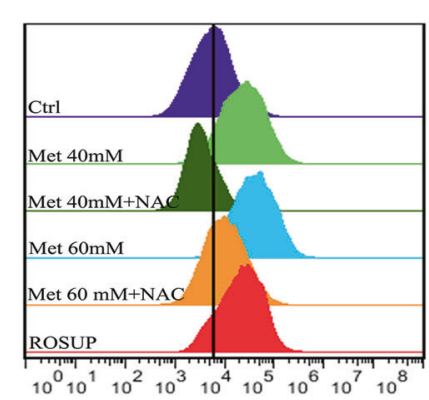

$\mathbf{F}$

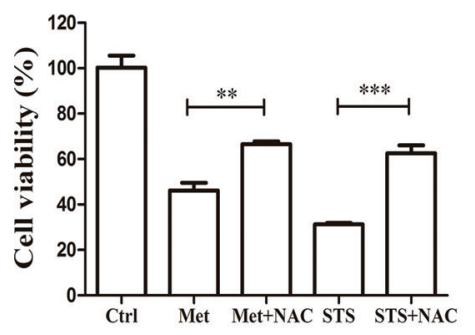

\section{FITC}

Fig. 2 Met induces ROS-dependent apoptosis in HCT116 cells. A The percentage of apoptotic cells quantitatively analyzed by using flow cytometry for the cells stained by Annexin-V/PI for at least 10,000 events per sample. B Level of cleaved-caspase-3, cleaved-caspase-8, and cleaved-caspase- 9 assessed by western blotting for the cells treated with Met for $24 \mathrm{~h}$. C Western blotting analysis on the Met-induced PARP cleavage. D Z-VAD inhibited Met-induced cytotoxicity. E Met or STS induced a significantly increased intracellular ROS. F NAC significantly inhibited Met-induced cytotoxicity. All data are expressed with the mean \pm SEM of three independent experiments. ${ }^{* *} p<0.01$ and ${ }^{* * *} p<0.001$ compared with the control group.

Met induces apoptosis by relieving the binding of Mcl-1 to Bak Antiapoptotic proteins (Bcl-2, Bcl-xL, Mcl-1) inhibit apoptosis by binding to proapoptotic proteins [16]. HCT116 cells exhibit high expression of $\mathrm{Mcl}-1$ and $\mathrm{BCl}-\mathrm{xL}$ [15]. Co-immunoprecipitation analysis showed that $\mathrm{Mcl}-1$ strongly bound to Bak, while $\mathrm{Bcl}-\mathrm{xL}$ weakly bound to Bak (Fig. 4A). Western blotting analysis showed that Met decreased $\mathrm{Mcl}-1$ expression, but elevated $\mathrm{Bcl}-\mathrm{xL}$ expression (Fig. 4B). However, overexpression of Mcl-1 instead of $\mathrm{BCl}-2 / \mathrm{BCl}-\mathrm{xL}$ significantly inhibited Met-induced loss of mitochondrial membrane potential (Fig. 4C), indicating that Mcl-1 instead of $\mathrm{BCl}-\mathrm{xL}$ plays a key inhibitory role in Met-induced apoptosis.

Quantitative FRET measurements were performed for the living cells co-expressing CFP-Mcl-1 and YFP-Bak (Fig. 4D). Figure 4D also shows the corresponding $E_{D}-R c$ plots from 109 cells. The two binding curves were saturable when $R c$ was larger than 2, and the 0.306 of $E_{D \max }$ in Met-treated cells was smaller than the 0.470 of $E_{D \max }$ in control cells, indicating that Met unlocked the binding of Mcl-1 to Bak. However, the $E_{D \max }(0.311, n=99)$ between CFP-Bcl$\mathrm{xL}$ and YFP-Bak in control cells was similar to $E_{D \max }(0.295, n=104)$ in Met-treated cells (Fig. 4E), indicating that Met has no effect on the binding of $\mathrm{BCl}-\mathrm{xL}$ to Bak.

\section{Bim is the key BH3-only protein to initiate Met-induced apoptosis}

$\mathrm{BH} 3-$ only proteins including Bim, $\mathrm{tBid}$, and Puma can directly activate Bak [16]. Western blotting analysis showed that Met promoted the expression of Bim, but did not cleave Bid into tBid and did not influence the expression of Puma and Bad (Fig. 5A). Only silencing
Bim significantly inhibited Met-induced loss of mitochondrial membrane potential (Fig. 5B), suggesting that Bim plays an important role in the Met-induced apoptosis. Co-immunoprecipitation analysis showed that Bim weakly bound to Bak in normal cells, and Met promoted the binding of Bim to Bak (Fig. 5C). Western blotting analysis showed that Met enhanced the AMPK phosphorylation (Fig. 5D), and compound C inhibited Met-induced Bim upregulation (Fig. 5E), indicating that Met activates AMPK to upregulates Bim.

Next, in order to evaluate the effect of Met on the binding of Mcl-1 to Bim, quantitative FRET measurements were used to measure the $E_{D}$ between CFP-Mcl-1 and YFP-Bim in 101 control cells and 106 Met-treated cells, respectively (Fig. 5F). The two binding curves were saturable when $R c$ was larger than 2, and the 0.3316 of $E_{D \max }$ in Met-treated cells was smaller than the 0.4456 of $E_{D \max }$ in control cells, indicating that Met unlocked the binding of Mcl-1 to Bim. Similarly, quantitative FRET measurements were performed for the living cells co-expressing CFP-Bak and YFP-Bim, and Fig. $5 \mathrm{G}$ shows the corresponding $E_{D}-R c$ plots from 92 control cells and 91 Met-treated cells. The two binding curves were saturable when $R c$ was larger than 1.0, and the 0.224 of $E_{D \max }$ between CFP-Bak and YFP-Bim in Met-treated cells was smaller than the 0.142 of $E_{D \max }$ in control cells, indicating that Met promoted the binding of Bim to Bak, which may activate Bak.

\section{DISCUSSION}

In this report, we dissected the molecular mechanism of Metinduced apoptosis in HCT116 cells. To our best knowledge, this is 
A

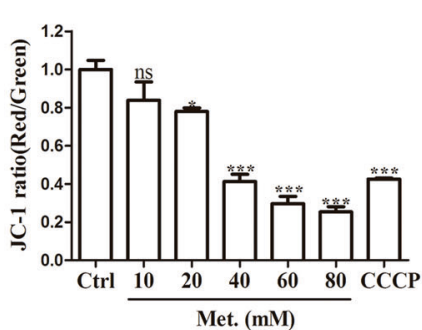

D

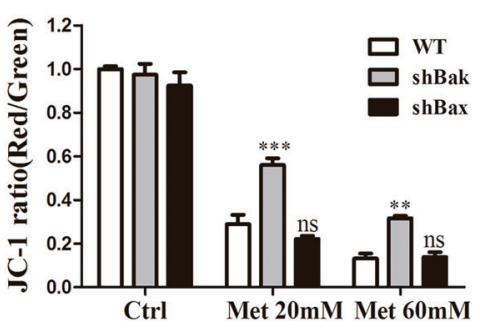

B

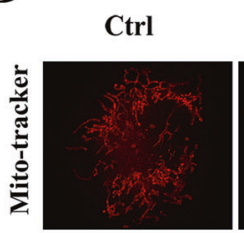

$\mathbf{E}$

GFP-Cyt.c

Mito-tracker

Merge
C

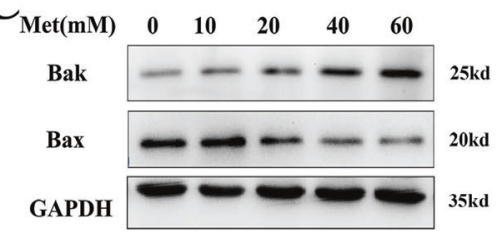

$\mathbf{F}$
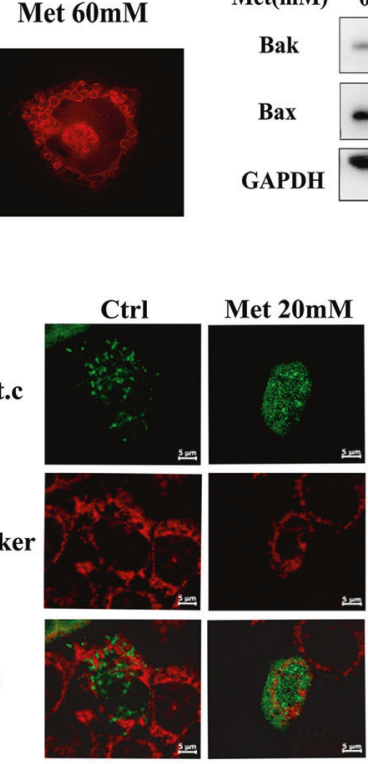
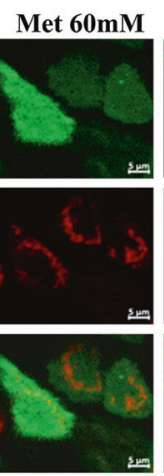

STS
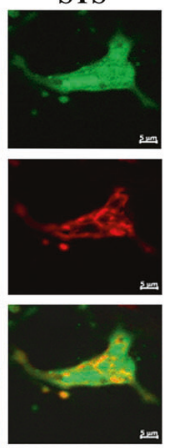

CFP-Bak+YFP-Bak
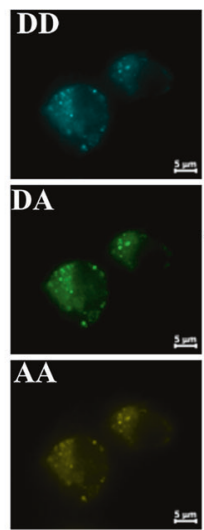
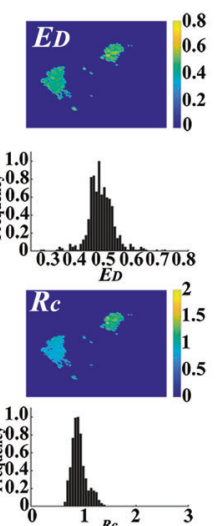

\section{CFP-Bak+YFP-Bak +Met}

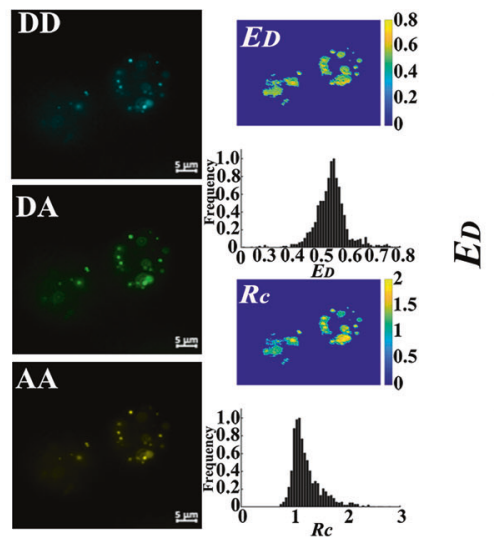

CFP-Bak+YFP-Bak

1.0 CFP-Bak+YFP-Bak+Met

过

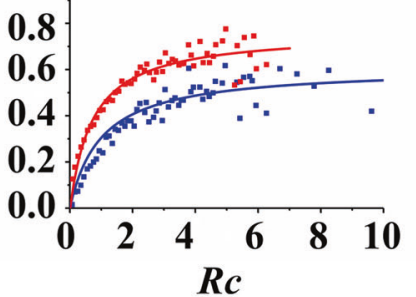

Fig. 3 Bak instead of Bax plays a key role in Met-induced apoptosis. A Met-induced dose-dependent loss of mitochondrial membrane potential $\left(n=60,{ }^{* * *} p<0.001\right.$ compared with the control group). B Met-induced mitochondrial swelling. Cells were stained with Mito-tracker Deep Red staining after treatment with $60 \mathrm{mM}$ Met for $24 \mathrm{~h}$. C Met upregulated Bak and downregulated Bax by western blotting analysis. Cells were treated with Met for $24 \mathrm{~h}$. D Silencing Bak instead of Bax inhibited Met-induced loss of mitochondrial membrane potential $(n=60$, ** $p<$ $0.01 ;{ }^{* * *} p<0.001$ compared with the WT group). E Met-induced release of cytochrome $c$ from mitochondria. Cells expressing GFP-cytochrome c were imaged. F Quantitative FRET measurements in living cells co-expressing CFP-Bak and YFP-Bak and the corrresponding ED-RC plot from at least 108 cells. All data are expressed with the mean \pm SEM of three independent experiments.

the first published report demonstrating that Met induces caspase- and ROS-dependent apoptosis dominantly via a Bakmediated intrinsic pathway, in which BH3 only protein Bim, as a key initiator, unlocks the binding of $\mathrm{Mcl}-1$ to Bak and then directly activates Bak.

Our observations that silencing Bak but not Bax by shRNA significantly prevented Met-induced loss of mitochondrial membrane potential (Fig. 3D) demonstrate that Bak, but not Bax, plays a key role in Met-induced apoptosis, which is further verified by Met-increased Bak homo-oligomerization (Fig. 3F). Inactive Bax is usually distributed in the cytoplasm and translocates to the mitochondria when it receives an apoptotic signal, while Bak is located in the mitochondrial membrane [24]. Microscopic imaging of cells expressing CFP-Bax showed that Met did not induce Bax translocation from cytosol to mitochondrion (data not shown), which also suggests that Bax is not involved in Met-induced apoptosis. It is generally considered that Bax plays a major role in the intrinsic apoptotic pathway, and Bak is an auxiliary role
$[25,26]$. However, recent publications reported that Bak plays a key role in apoptosis induced by artesunate, actinomycin D and tumor necrosis factor-related apoptosis-inducing ligand (TRAIL), respectively $[27,28]$. We found that Met-induced Bax upregulation and Bcl-xL down-regulation in MCF-7 and A549 cells (Fig. S3) support previous notion that Bax and $\mathrm{Bcl}-\mathrm{xL}$ plays an important role in metformin-induced apoptosis [29, 30]. However, our observations that Bak instead of Bax plays a key role in Metinduced apoptosis in HCT116 cells.

The findings that overexpression of $\mathrm{Mcl}-1$ instead of $\mathrm{Bcl}-2$ or $\mathrm{Bcl}-$ $\mathrm{xL}$ inhibited Met-induced loss of mitochondrial membrane potential (Fig. 4C) demonstrate that $\mathrm{Mcl}-1$ plays an important role in inhibiting Met-induced apoptosis, which is further verified by the findings that Met unlocked the binding of Bak to Mcl-1 but did not affect the binding of Bak to Bcl-xL (Fig. 4D, E). Bcl-xL and Mcl-1 can efficiently inhibit Bak activation $[16,31]$. The dynamic balance between $\mathrm{Mcl}-1 / \mathrm{BCl}-\mathrm{xL}$ and Bak will be broken by apoptosis stimuli [16]. However, in some cases, $\mathrm{Mcl}-1$ and $\mathrm{BCl}-\mathrm{xL}$ do not work 

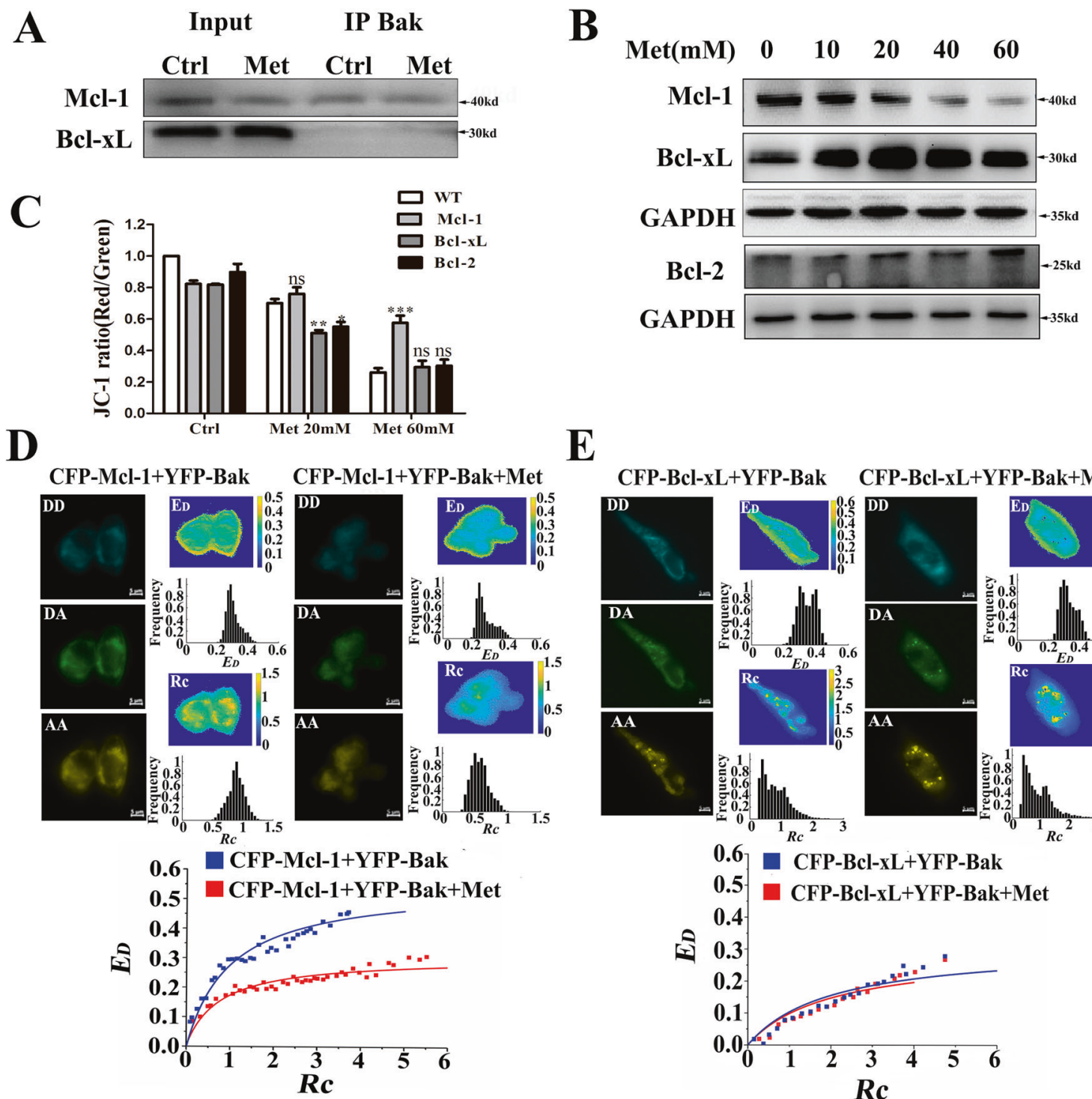

$\mathbf{E}$
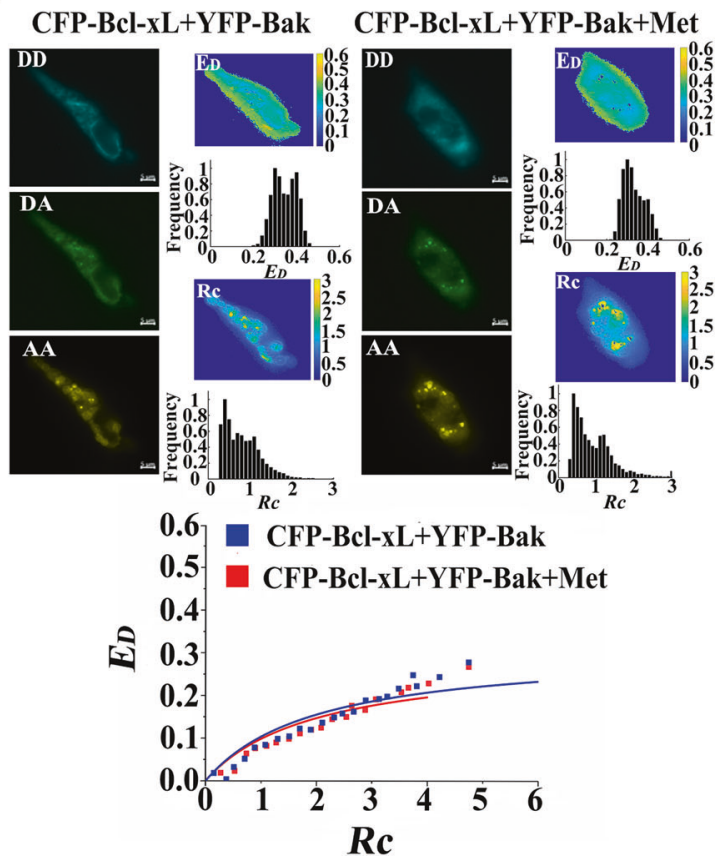

Fig. 4 Met induces apoptosis by relieving the binding of $\mathrm{Mcl}-1$ to Bak. A Co-immunoprecipitation analysis on the binding of $\mathrm{Mcl}-1 / \mathrm{Bcl}-\mathrm{xL}$ to Bak. B Western blotting analysis on the $\mathrm{Mcl}-1$ and $\mathrm{Bcl}-\mathrm{xL}$ expression after Met treatment for $24 \mathrm{~h}$. C Overexpression Mcl-1 instead of Bcl-2 or $\mathrm{BCl}-\mathrm{xL}$ inhibited Met-induced loss of mitochondrial membrane potential $\left(n=53,{ }^{* * *} p<0.001\right.$ compared with the WT group). D Quantitative FRET measurements for living cells co-expressing CFP-Mcl-1 and YFP-Bak, and the corresponding $E_{D}-R c$ plot from at least 109 cells. E Quantitative FRET measurements for living cells co-expressing CFP-BCl-xL and YFP-Bak, and the corresponding $E_{D}-R c$ plot from at least 99 cells. All data are expressed with the mean \pm SEM of three independent experiments.

at the same time $[16,21]$. For example, Met-induced apoptosis in OVCAR-3 and OVCAR-4 cells by downregulating $\mathrm{BCl}-2$ and $\mathrm{BCl}-\mathrm{xL}$ [20]. In MYC-dependent apoptosis, Met acted by inhibiting $\mathrm{Bcl}-2$ and $\mathrm{BCl}-\mathrm{xL}$ instead of $\mathrm{Mcl}-1$ [32]. However, Met promoted cell death by downregulating the expression of $\mathrm{Mcl}-1$ under low glucose conditions [21]. Our live-cell FRET imaging that Met unlocked the binding of $\mathrm{Mcl}-1$ to Bak (Fig. 4D) and Met-increased Bak homo-oligomerization (Fig. 3F) firmly demonstrate that Met induces Bak-mediated apoptosis by inhibiting $\mathrm{Mcl}-1$ in HCT116 cells.

Our observations that silencing Bim instead of Bid, Puma and Bad inhibited Met-induced loss of mitochondrial membrane potential (Fig. 5) demonstrate that Bim is the key BH3-only protein to initiate Met-induced apoptosis, which is further verified by the findings that Met unlocked the binding of Mcl-1 to Bim and enhanced the binding of Bim to Bak (Fig. 4D, E). Bid, Bim and Puma are capable of directly activating Bax/Bak [33]. Puma exhibits a weak direct interaction with Bax/Bak compared with
Bim and tBid [34, 35]. tBid is generally considered to be has stronger proapoptotic ability than Bim and plays an important role in apoptosis induced by many kinds of stimuli. The active caspase8 cleaves Bid into tBid to activate Bax/Bak $[36,37]$. Although Metinduced caspase-8 activation (Fig. $3 \mathrm{~B}$ ), Met did not cleave Bid into tBid (Fig. 5A), indicating that Bid may not be involved in Metinduced apoptosis, which is further verified by the finding that silencing Bid did not prevent Met-induced apoptosis. In addition, several studies reported that TRAIL induced caspase-8 activation and Bid cleavage, but tBid was unable to induce Bak oligomerization and MOMP [38]. Our findings that Met enhanced AMPK phosphorylation and compound $C$ inhibited Met-induced Bim upregulation (Fig. 5D, E) support previous notion that AMPK plays a crucial role in Bim upregulation [39]. Our data that Met not only decreases $\mathrm{Mcl}-1$ expression (Fig. $4 \mathrm{~B}$ ) and relieves the binding of Mcl-1 to Bim (Fig. 5F) but also increases Bim expression (Fig. 5A) and enhances the binding of Bim to Bak (Fig. 5G) firmly demonstrate the key role of Bim in Met-induced apoptosis. 
A

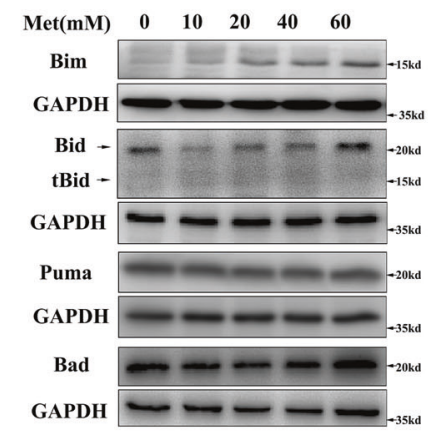

B

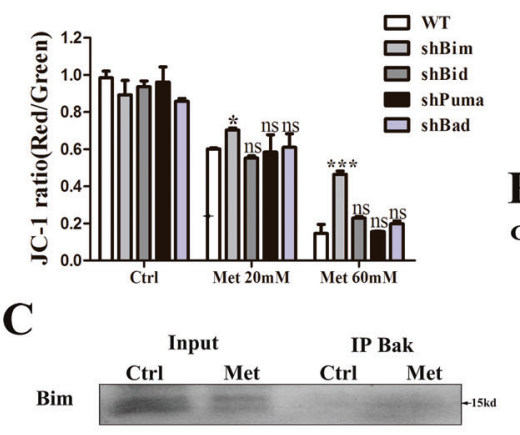

D

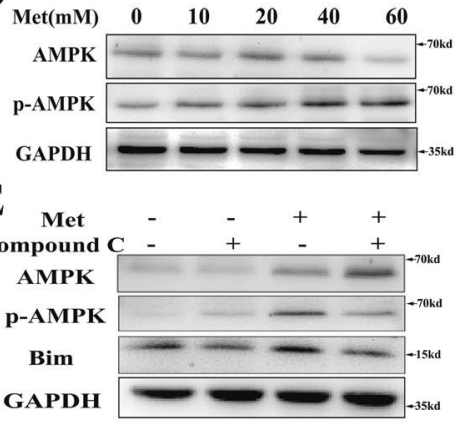

F
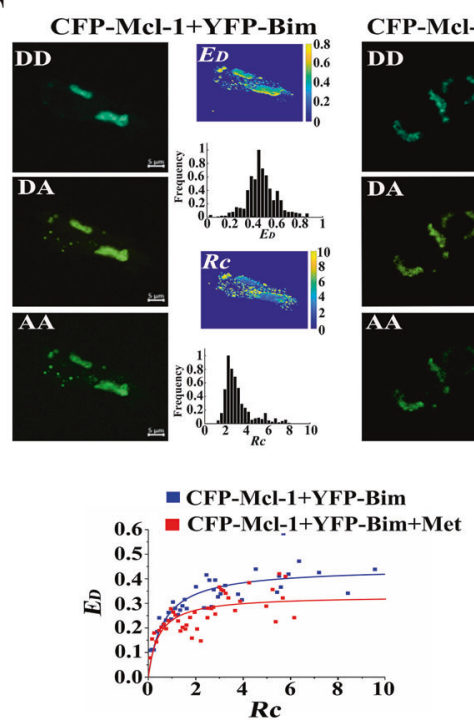

G

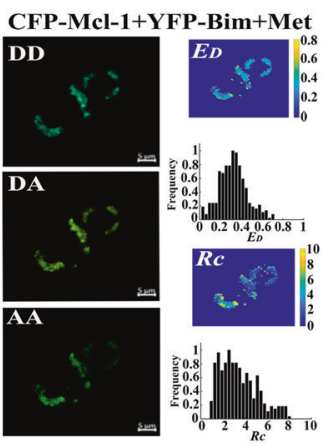

CFP-Bak+YFP-Bim+Met
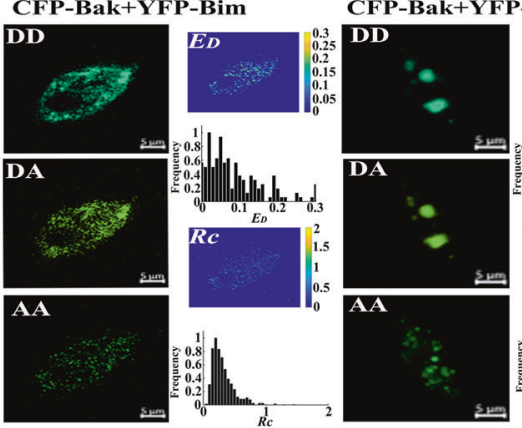
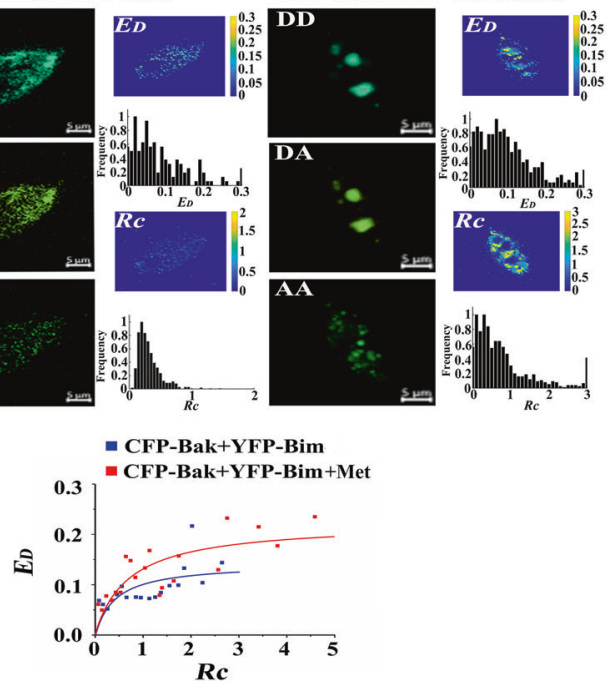

Fig. 5 Bim is the key BH3-only protein to initiate Met-induced apoptosis. A Western blotting analysis on Puma, Bim, Bid and Bad expression after Met treatment for $24 \mathrm{~h}$. B Effects of silencing Puma, Bim, Bid, and Bad respectively on the Met-induced loss of mitochondrial membrane potential $\left(n=38,{ }^{*} p<0.05\right.$ and ${ }^{* * *} p<0.001$ compared with the WT group). C Co-immunoprecipitation analysis on the binding of Bim to Bak. D Met enhanced the AMPK phosphorylation. E Compound C inhibited Met-induced AMPK phosphorylation and Bim expression. F Quantitative FRET measurements for living cells co-expressing CFP-Mcl-1 and YFP-Bim, and the corresponding $E_{D}-R c$ plot from at least 100 cells. G Quantitative FRET measurements for living cells co-expressing CFP-Bak and YFP-Bim, and the corresponding $E_{D}-R c$ plot from at least 90 cells. All data are expressed with the mean \pm SEM of three independent experiments.

Based on our data, we summarize the Met-induced apoptotic pathway in HCT116 cells (Fig. 6). Met induces Bak-mediated apoptosis in ROS- and caspase-dependent fashion. Met induces the phosphorylation and activation of AMPK to upregulate Bim. Meanwhile, Met unlocks the binding of Mcl-1 to Bak, and promotes the binding of Bim to Bak, which leads to Bak activation and homo-oligomerization, leading to MOMP and subsequent cytochrome c release and caspase activation. However, how Bim participates in the molecular mechanism by which Met inhibits the interaction between Bak and Mcl-1is unknown, which will be the task of future investigations.

\section{MATERIALS AND METHODS}

\section{Materials}

Met was purchased from Solarbio (Beijing, China). Staurosporine (STS), Caspase-3/8/9 Assay Kit, ROS Assay Kit and JC-1 Assay Kit were provided by Beyotime (Shanghai, China). Anti-PARP (9532), anti-Bak (12105), antiBax (5023), anti-BCl-xL (2764), anti-Mcl-1 (94296), anti-Bim (2933), antiBad (9268), anti-Puma (98672), anti-AMPK (5831), anti-p-AMPK (8208), anti-Cleaved Caspase-3 (9664), anti-Cleaved Caspase-8 (8592), antiCaspase-9 (9502) Rabbit monoclonal antibody, and anti-Bid (8762) Mouse monoclonal antibody were purchased from Cell Signaling Technology (Danvers, MA, USA). Anti-GAPDH (sc-47724) Mouse monoclonal antibody was obtained from Santa Cruz (Texas, USA). Luciferase Mycoplasma Detection Kit and anti- $\beta$-tubulin (HC101) Mouse monoclonal antibody were purchased from Transgen Biotech (Beijing, China). Turbofect ${ }^{\mathrm{TM}}$ transfection regent and Mito-tracker Deep Red dye were obtained from ThermoFisher Scientific (Massachusetts, USA). Cell counting Kit-8 (CCK-8) and Alexa Fluor 488 annexin V/Dead Cell Apoptosis Kit were purchased from Dojindo (Kyushu, Japan). Acetylcysteien (NAC), Z-VAD-(OMe)-FMK (Z-VAD) and Compound $C$ were purchased from MedChemExpress (New Jersey, USA). Hoechst 33258 dye and PI dye were obtained from G-clone (Beijing, China).

\section{Constructs}

The CFP-YFP dimers including YFP-G10-CFP and YFP-G40-CFP were kindly provided by Christian Wahl-Schott [40]. Plasmids encoding the CFP-BCl-xL was kindly supplied by A. P. Gilmore [41]. pTOPO-Mcl-1 (\#21065), ECFP-Bak (\#31501) plasmids were purchased from Addgene Company. YFP-Bim was synthesized by Gene Create Company. YFP-Bak, CFP-Mcl-1 plasmids and sh Bax, shPuma, shBad were constructed as previously described [27, 42-45]. The silencing plasmids of Bak, Bim and Bid (\#p31235, \#p31337, \#p31234) were designed and synthesized by Miaoling Biotech Company (Wuhan, China). Sequences for shRNA were: Bak shRNA 1\#, 5'-GTTTGTGGTACG AAGATTCAAGAGATCTTCGTACCACAAACTGGCCTTIITTG-3'; Bak shRNA 2\#, 5'-GTTTGTGGTACGAAGATTCAAGAGATCTTCGTACCACAAACTGGCCTITITा G-3', Bak shRNA 3\#,5'-GTTTGTGGTACGAAGATTCAAGAGATCTTCG;TACCAC AAACTGGCCTIITITG-3'; Bim shRNA 1\#,5'-GCAACCTTCTGATGTAAGTTCTTC 


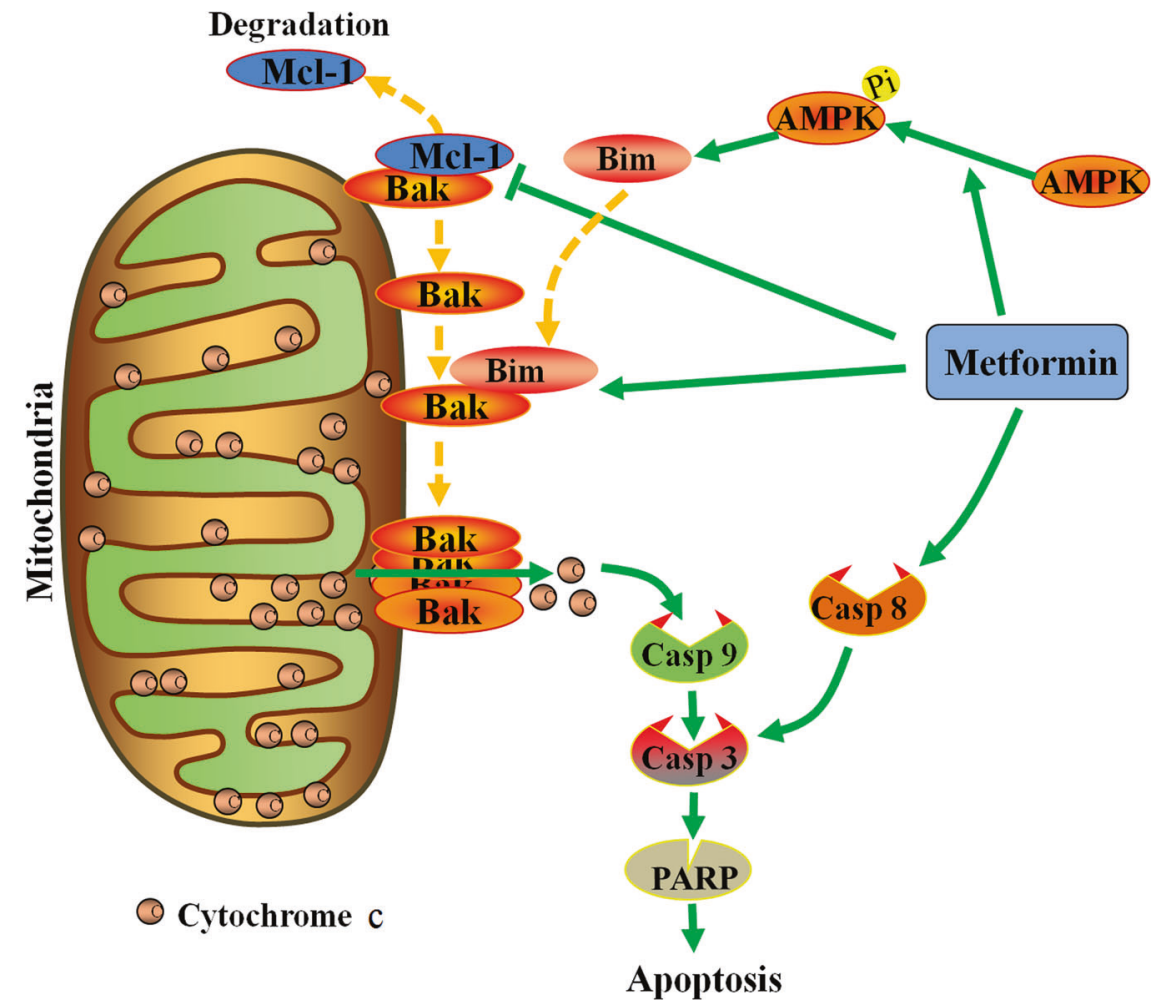

Fig. 6 Schematic diagram of the molecular mechanism of Met against HCT116 cells. Met induces the phosphorylation and activation of AMPK to up-regulate Bim. Meanwhile, Met unlocks the binding of Mcl-1 to Bak, and promotes the binding of Bim to Bak, which leads to Bak activation and homo-oligomerization, leading to cytochrome $\mathrm{c}$ release and caspase activation of downstream apoptosis signaling pathway.

AAGAGAGAACTTACATCAGAAGGTTGCTT-3'; Bim ShRNA 2\#,5'-GGCCTT CAACCACTATCTCAGTTCAAGAGACTGAGATAGTGGTTGAAGGCCTT-3'; Bim ShRNA 3\#,5'-GCTACCTCCCTACAGACAGATTCAAGAGATCTGTCTGTAGGGAG GTAGCC- $3^{\prime}$. All constructs used in this work were confirmed by DNA sequencing and enzyme digestion diagnosis.

\section{Cell lines and cell culture}

The HCT116 cells, MCF-7 cells, and A549 cells were obtained from the tumor cell bank of the Chinese Academy of Medical Science. The cells were cultured in Medium DMEM containing 10\% fetal bovine serum, $100 \mathrm{U} / \mathrm{mL}$ penicillin $\mathrm{G}$ and $100 \mu \mathrm{g} / \mathrm{mL}$ streptomycin in a humidified atmosphere of $5 \% \mathrm{CO}_{2}$ at $37^{\circ} \mathrm{C}$. All experiments were performed during the exponential phase of cell growth. Stocks of authenticated cell lines were cryopreserved, all cell lines were in early passages and maintained in culture for $<3$ months, tested regularly for mycoplasma contamination using luciferase mycoplasma detection kit.

\section{Cell viability assay}

The inhibitory effect of Met on the viability of HCT116 cells were measured by the CCK-8 assay according to the protocol described by the manufacturer. In brief, cells were seeded in 96-well plates at a density of $1.0 \times 10^{5}$ cells/well with a volume of $100 \mu \mathrm{L}$ and grown at $37^{\circ} \mathrm{C}$ for $24 \mathrm{~h}$, then treated with different concentrations of Met for $24 \mathrm{~h}$. Subsequently, $10 \mu \mathrm{L}$ of CCK-8 solution was added to each well and the plates were incubated at $37^{\circ} \mathrm{C}$ for $1 \mathrm{~h}$, after which the optical density of each well was determined at $570 \mathrm{~nm}$ using a microplate reader. All the experiments were repeated three times independently. The half maximal inhibitory concentration (IC50) values of Met in HCT116 cells were calculated.

\section{Characterization of cell death}

The morphology of nucleus was analyzed by Hoechst /PI double staining. In brief, cells were grown on the confocal dishes for $24 \mathrm{~h}$. After being treated with indicated treatments, the cells were incubated with $20 \mu \mathrm{g} / \mathrm{mL}$ Hoechst for $30 \mathrm{~min}$ and then with $10 \mu \mathrm{g} / \mathrm{mL}$ PI for $15 \mathrm{~min}$ at $37^{\circ} \mathrm{C}$ in the dark. Then, the cells were washed once with PBS and observed with fluorescence microscope.

\section{Flow cytometry}

Identification of apoptotic cells was achieved by Annexin V-FITC/PI staining. HCT116 cells $\left(2.0 \times 10^{5}\right.$ cells $\left./ \mathrm{mL}\right)$ were seeded in six-well plates and treated with different concentrations of Met for $24 \mathrm{~h}$. Annexin V-FITC/ PI staining was performed according to the instructions provided by the manufacturer. $1.0 \times 10^{6}$ cells per sample was collected by $0.2 \%$ Trypsin, washed twice with PBS, cells were resuspended with $1 \times$ binding buffer at a density of $1.0 \times 10^{6}$ cells $/ \mathrm{mL}$. Annexin V-FITC and PI $(5 \mu \mathrm{L})$ were added to cell suspensions $(100 \mu \mathrm{L})$ and further incubated for $15 \mathrm{~min}$ at room temperature in the dark. Stained cells were diluted with $1 \times$ binding buffer and immediately analyzed by flow cytometry. All experiments were repeated three times.

\section{Caspase activity assay}

The HCT116 cells were placed in 6 -well plates at $2 \times 10^{5}$ cells/well. According to the manufacturer's protocol, treated cells were lysed with lysis buffer for $15 \mathrm{~min}$ on ice following washing with cold PBS, the supernatants collected and protein concentration determined by BCA protein assay. Incubate the mixture composed of $10 \mu \mathrm{L}$ of cell lysate, $80 \mu \mathrm{L}$ of reaction buffer and $10 \mu \mathrm{L}$ of $2 \mathrm{mM}$ caspase-3 substrate in 96-well microtiter plates at $37^{\circ} \mathrm{C}$ for $4 \mathrm{~h}$, and the activity of caspase- 3 were quantified in the samples with enzyme-labeling instrument at an absorbance of $405 \mathrm{~nm}$. Relative caspase activity was calculated as the emission ratio between the treated cells to the untreated cells.

\section{Detection of intracellular ROS}

Intracellular ROS was detected by means of an oxidation-sensitive fluorescent probe (DCFH-DA). After treatment with Met for $24 \mathrm{~h}$, cells were washed twice in phosphate-buffered saline (PBS), then incubated with $10 \mu \mathrm{M} / \mathrm{L}$ DCFH-DA at $37^{\circ} \mathrm{C}$ for $30 \mathrm{~min}$ according to the manufacturer's instructions. DCFH-DA was deacetylated intracellularly by nonspecific esterase, which was further oxidized by ROS to the fluorescent compound 2,7-dichlorofluorescein (DCF). DCF fluorescence was detected by flow cytometer.

\section{Western blotting analysis}

For the extraction of whole proteins, cells were collected and washed twice with pre-cold PBS. Cells were then treated with RIPA lysis buffer containing 
protease inhibitors to obtain cell lysates. Next, the lysates were sonicated and centrifuged at $13,000 \mathrm{rpm}$ for $10 \mathrm{~min}$. Supernatant was recovered and quantified by the BCA protein assay, and stored at $-20^{\circ} \mathrm{C}$. The proteins were separated by $12 \%$ SDS-PAGE, electrophoretically transferred onto $0.22 \mu \mathrm{m}$ polyvinylidene difluoride membranes. After blocking with $5 \%$ skimmed milk at room temperature for $2 \mathrm{~h}$, the membranes was incubated overnight at $4{ }^{\circ} \mathrm{C}$ with antibodies: rabbit anti-human Bak antibody (1: 1 000). After washing three times with TBST, the membranes were incubated with HRP-labeled goat anti-rabbit polyclonal antibody $(1: 10,000)$ at room temperature for $2 \mathrm{~h}$. After washing with TBST buffer, the immunoreactive bands were visualized by enhanced chemiluminescence chemistry substrate.

\section{Co-Immunoprecipitation}

HCT116 cells were lysed in RIPA buffer with protease inhibitor for $5 \mathrm{~min}$, centrifuged at $13,000 \mathrm{rpm}$ for $10 \mathrm{~min}$ at $4{ }^{\circ} \mathrm{C}$ and the supernatants were collected of total lysates, then immunoprecipitated with anti-Bak antibody at room temperature for $2 \mathrm{~h}$. Collected the Protein-A/G magnetic beads, the beads were washed with RIPA buffer three times. All protein samples were boiled for $5 \mathrm{~min}$ in standard loading buffer with $20 \%$ loading buffer before western blotting detection.

\section{Quantitative FRET imaging}

Quantitative FRET imaging was performed on a fluorescence microscope (Axio Observer 7, Carl Zeiss, Oberkochen, Germany) as previously described [17]. Donor-centric FRET efficiency $\left(E_{D}\right)$ and acceptor-donor ratio $(R c)$ were measured by using FRET method just as described previously $[46,47]$.

\section{$E_{D}$ saturation assay}

Cells were co-transfected with $200 \mathrm{ng}$ of plasmid encoding CFP-tagged protein and various concentrations $(200,400,600$, and $800 \mathrm{ng}$, respectively) of plasmid encoding YFP-tagged protein. In general, only the cells with fluorescence signals at least three times higher than backgrounds and $<60,000$ (the saturation value of fluorescence signal is 65,535 ) were chosen for analysis. $E_{D}$ values were distributed into bins of different sizes according to concentration ratio of total acceptor-to-donor $(R c)$ and plotted against Rc. The saturation binding curves were fitted using Origin with the function: $E_{D}=E_{D \max } \times R c /\left(K_{d}+R c\right)$. $E_{D \max }$ is the maximum $E_{D}$ corresponding to saturation of donor binding sites by an acceptor and $K_{d}$ is the relative equilibrium dissociation constant $[43,48]$.

\section{Statistical analysis}

The $p$ values for datasets were analyzed by unpaired Student's $T$ test, $p<$ 0.05 was defined as statistical significance. Statistical and graphic analyses were done using the software Graph Pad Prism 5 and Origin 8.0.

\section{DATA AVAILABILITY}

The datasets used and/or analyzed during the current study are available from the corresponding author on reasonable request.

\section{REFERENCES}

1. Ritwika M, Chowdhury TA. Metformin in cancer. Diabetes Res Clin Pract 2018;143:409-19.

2. Pernicova I, Korbonits M. Metformin-mode of action and clinical implications for diabetes and cancer. Nat. Rev Endocrinol 2014;10:143-56.

3. Krishan S, Richardson DR, Sahni, S. Adenosine monophosphate-activated kinase and its key role in catabolism: structure, regulation, biological activity, and pharmacological activation. Mol Pharmacol 2015;87:363-77.

4. Faubert B, Vincent EE, Poffenberger, MC, Jones RG. The AMP-activated protein kinase (AMPK) and cancer: many faces of a metabolic regulator. Cancer Lett 2015;356:165-70.

5. Key TJ, Appleby PN, Reeves GK, Roddam AW. Insulin-like growth factor 1 (IGF1), IGF binding protein 3 (IGFBP3), and breast cancer risk: pooled individual data analysis of 17 prospective studies. Lancet Oncol 2010;11:530-42.

6. Abo-Elmatty DM, Ahmed EA, Tawfik MK, Helmy SA. Metformin enhancing the antitumor efficacy of carboplatin against Ehrlich solid carcinoma grown in diabetic mice: effect on IGF-1 and tumoral expression of IGF-1 receptors. Int Immunopharmacol 2017;44:72-86.
7. Sung H, Ferlay J, Siegel RL, Laversanne M, Soerjomataram I, Jemal A, et al. Global cancer statistics 2020: GLOBOCAN estimates of incidence and mortality worldwide for 36 cancers in 185 countries. CA Cancer J Clin 2021;71:209-49.

8. Siegel RL, Miller KD, Goding Sauer A, Fedewa SA, Butterly LF, Anderson JC, et al. Colorectal cancer statistics, 2020. CA Cancer J Clin 2020;70:145-64.

9. Poston GJ, Figueras J, Giuliante F, Nuzzo G, Sobrero AF, Gigot JF, et al. Urgent need for a new staging system in advanced colorectal cancer. $J$ Clin Oncol 2009;26:4828-33.

10. Chu E. An update on the current and emerging targeted agents in metastatic colorectal cancer. Clin Colorectal Cancer. 2012;11:1-13.

11. Zhang L, Yu J. Role of apoptosis in colon cancer biology, therapy, and prevention. Curr Colorectal Cancer Rep. 2013;9:10.

12. Lee JH, Kim TI, Jeon SM, Hong SP, Cheon JH, Kim WH. The effects of metformin on the survival of colorectal cancer patients with diabetes mellitus. Int $J$ Cancer. 2012;131:752-9.

13. Lee, MS, Hsu, CC, Wahlqvist, ML, Tsai, HN, Chang, YH, Huang, YC. Type 2 diabetes increases and metformin reduces total, colorectal, liver and pancreatic cancer incidences in Taiwanese: a representative population prospective cohort study of 800,000 individuals. BMC Cancer. 2011;11:20.

14. Tomimoto A, Endo $H$, Sugiyama M, Fujisawa T, Hosono $K$, Takahashi $H$, et al. Metformin suppresses intestinal polyp growth in ApcMin/+ mice. Cancer Sci. 2008;99:2136-41.

15. Placzek WJ, Wei J, Kitada S, Zhai D, Reed JC, Pellecchia M. A survey of the antiapoptotic $\mathrm{BCl}-2$ subfamily expression in cancer types provides a platform to predict the efficacy of $\mathrm{Bcl}-2$ antagonists in cancer therapy. Cell Death Dis. 2010;1:18.

16. Singh R, Letai A, Sarosiek K. Regulation of apoptosis in health and disease: the balancing act of BCL-2 family proteins. Nat Rev Mol Cell Biol. 2019;20:175-93.

17. Aranovich A, Liu Q, Collins T, Geng F, Dixit S, Leber B, et al. Differences in the mechanisms of proapoptotic $\mathrm{BH} 3$ proteins binding to $\mathrm{Bcl}-\mathrm{XL}$ and $\mathrm{Bcl}-2$ quantified in live MCF-7 cells. Mol Cell. 2012;45:754-63.

18. Youle RJ, Strasser A. The BCL-2 protein family: opposing activities that mediate cell death. Nat Rev Mol Cell Biol. 2008;9:47-59.

19. Sahin E, Eraslan Sahin M, Dolanbay M, Ozcelik B, Akgun H, Saatci C. Induction of apoptosis by metformin and progesterone in estrogen-induced endometrial hyperplasia in rats: involvement of the bcl-2 family proteins. Gynecol Endocrinol. 2018;34:433-6.

20. Yasmeen A, Beauchamp MC, Piura E, Segal E, Pollak M, Gotlieb WH. Induction of apoptosis by metformin in epithelial ovarian cancer: involvement of the $\mathrm{BCl}-2$ family proteins. Gynecol Oncol. 2011;121:492-8.

21. Elgendy M, Cirò M, Hosseini A, Weiszmann J, Mazzarella L, Ferrari E, et al. Combination of hypoglycemia and metformin impairs tumor metabolic plasticity and growth by modulating the PP2A-GSK3ß-MCL-1 axis. Cancer Cell. 2019;35:798-815.

22. Smith S. The world according to PARP. Trends Biochem Sci. 2001;26:174-9.

23. Warren CFA, Wong-Brown MW, Bowden NA. BCL-2 family isoforms in apoptosis and cancer. Cell Death Dis. 2019;10:177.

24. Gélinas $C$, White $\mathrm{E}$. BH3-only proteins in control: specificity regulates MCL-1 and BAK-mediated apoptosis. Genes Dev. 2005;19:1263-8.

25. Karlberg M, Ekoff M, Labi V, Strasser A, Huang D, Nilsson G. Pro-apoptotic Bax is the major and Bak an auxiliary effector in cytokine deprivation-induced mast cell apoptosis. Cell Death Dis. 2010;1:20.

26. Wendt J, von Haefen C, Hemmati P, Belka C, Dörken B, Daniel PT. TRAIL sensitizes for ionizing irradiation-induced apoptosis through an entirely Bax-dependent mitochondrial cell death pathway. Oncogene. 2005;24:4052-64.

27. Zhou C, Pan WF, Wang XP, Chen TS. Artesunate induces apoptosis via a Bakmediated caspase-independent intrinsic pathway in human lung adenocarcinoma cells. J. Cell Physiol. 2012;227:3778-86.

28. Neise D, Graupner V, Gillissen BF, Daniel PT, Schulze-Osthoff K, Jänicke RU, et al. Activation of the mitochondrial death pathway is commonly mediated by a preferential engagement of Bak. Oncogene. 2008;27:1387-96.

29. Ma J, Guo Y, Chen S, Zhong C, Xue Y, Zhang Y, et al. Metformin enhances tamoxifen-mediated tumor growth inhibition in ER-positive breast carcinoma. BMC Cancer. 2014;14:172.

30. Malki A, Youssef A. Antidiabetic drug metformin induces apoptosis in human MCF breast cancer via targeting ERK signaling. Oncol. Res. 2011;19:275-85.

31. Cheng EH, Wei MC, Weiler S, Flavell RA, Mak TW, Lindsten T, et al. BCL-2, BCL-X(L) sequester $\mathrm{BH} 3$ domain-only molecules preventing BAX- and BAK-mediated mitochondrial apoptosis. Mol. Cell. 2001;8:705-11.

32. Haikala HM, Anttila JM, Marques E, Raatikainen T, Ilander M, Hakanen $H$, et al. Pharmacological reactivation of MYC-dependent apoptosis induces susceptibility to anti-PD-1 immunotherapy. Nat. Commun. 2019;10:932.

33. Minet E, Cosse JP, Demazy C, Raes M, Michiels C. Accumulation of the proapoptotic factor Bak is controlled by antagonist factor Mcl-1 availability. Apoptosis. 2006;11:1039-47. 
34. Ghiotto F, Fais F, Bruno S. BH3-only proteins: the death-puppeteer's wires. Cytom. A. 2010;77:11-21.

35. Chipuk JE, Green DR. PUMA cooperates with direct activator proteins to promote mitochondrial outer membrane permeabilization and apoptosis. Cell Cycle. 2009;8:2692-6.

36. Chipuk JE, Green DR. How do BCL-2 proteins induce mitochondrial outer membrane permeabilization? Trends Cell Biol. 2008;18:157-64.

37. Hockings $C$, Anwari K, Ninnis RL, Brouwer J, O'hely M, Evangelista M, et al. Bid chimeras indicate that most $\mathrm{BH} 3$-only proteins can directly activate Bak and Bax, and show no preference for Bak versus Bax. Cell Death Dis. 2015;6:105.

38. Son JK, Varadarajan S, Bratton SB. TRAIL-activated stress kinases suppress apoptosis through transcriptional upregulation of MCL-1. Cell Death Differ. 2010;17:1288-301.

39. Davila D, Connolly NM, Bonner H, Weisová P, Dussmann H, Concannon CG, et al. Two-step activation of FOXO3 by AMPK generates a coherent feed-forward loop determining excitotoxic cell fate. Cell Death Differ. 2012;19:1677-88.

40. Butz ES, Ben-Johny M, Shen M, Yang PS, Sang L, Biel M, et al. Quantifying macromolecular interactions in living cells using FRET two-hybrid assays. Nat. Protoc. 2016;11:2470-98.

41. Valentijn AJ, Metcalfe AD, Kott J, Streuli CH, Gilmore AP. Spatial and temporal changes in Bax subcellular localization during anoikis. J. Cell Biol. 2003; 162:599-612.

42. Wang Y, Su WH, Mai ZH, Yu S, Wang XP, Chen TS. Anti-apoptotic capacity of Mcl1 127 . Biochem Biophys. Res Commun. 2020;526:1042-8.

43. Yang FF, Qu WF, Du MY, Mai ZH, Wang B, Ma YY, et al. Stoichiometry and regulation network of $\mathrm{Bcl}-2$ family complexes quantified by live-cell FRET assay. Cell Mol. Life Sci. 2020;77:2387-406.

44. Gao WF, Xiao F, Wang X, Chen TS. Artemisinin induces A549 cell apoptosis dominantly via a reactive oxygen speciesmediated amplification activation loop among caspase-9, -8 and -3. Apoptosis. 2013;18:1201-13.

45. Qin GQ, Zhao CS, Zhang L, Liu H, Quan YY, Chai L, et al. Dihydroartemisinin induces apoptosis preferentially via a Bimmediated intrinsic pathway in hepatocarcinoma cells. Apoptosis. 2015;20:1072-86.

46. Hoppe A, Christensen K, Swanson JA. Fluorescence resonance energy transferbased stoichiometry in living cells. Biophys. J. 2002;83:3652-64.

47. Erickson MG, Alseikhan BA, Peterson BZ, Yue DT. Preassociation of calmodulin with voltage-gated $\mathrm{Ca}(2+)$ channels revealed by FRET in single living cells. Neuron. 2001;31:973-85.

48. Ben-Johny M, Yue DN, Yue DT. Detecting stoichiometry of macromolecular complexes in live cells using FRET. Nat. Commun. 2016;7:13709.

\section{ACKNOWLEDGEMENTS}

We thank Christian Wahl-Schott for providing YFP-G10-CFP and YFP-G40-CFP plasmids and Andrew P. Gilmore for providing CFP-Bax plasmid.

\section{AUTHOR CONTRIBUTIONS}

HC designed and performed experiments, analyzed data, and wrote the paper. BS performed the flow cytometry experiments, cell culture, and analyzed data. HS designed the MATLAB scripts. XF, GW, and LX performed the WB experiments. ZT and ZM analyzed the FRET data. QT and XC constructed the plasmids. HC, XW, and TC designed the study, planned experiments, and wrote the paper.

\section{FUNDING}

This work was supported by grants from the National Natural Science Foundation of China (NSFC) (Grant numbers: 61875056 and 62135003), the Science and Technology Program of Guangzhou (Grant number: 2019050001), The Scientific and Technological Plan of Guangdong Province, China (No. 2019B090905005), Natural Science Foundation of Guangdong Province (Grant number: 2021A1515011221), and the Combined Foundation of Guangzhou City and University (college) for basic research project (Grant number: 202102010039).

\section{COMPETING INTERESTS}

The authors declare no competing interests.

\section{ADDITIONAL INFORMATION}

Supplementary information The online version contains supplementary material available at https://doi.org/10.1038/s41420-021-00755-y.

Correspondence and requests for materials should be addressed to Xiaoping Wang or Tongsheng Chen.

Reprints and permission information is available at http://www.nature.com/ reprints

Publisher's note Springer Nature remains neutral with regard to jurisdictional claims in published maps and institutional affiliations.

Open Access This article is licensed under a Creative Commons Attribution 4.0 International License, which permits use, sharing, adaptation, distribution and reproduction in any medium or format, as long as you give appropriate credit to the original author(s) and the source, provide a link to the Creative Commons license, and indicate if changes were made. The images or other third party material in this article are included in the article's Creative Commons license, unless indicated otherwise in a credit line to the material. If material is not included in the article's Creative Commons license and your intended use is not permitted by statutory regulation or exceeds the permitted use, you will need to obtain permission directly from the copyright holder. To view a copy of this license, visit http://creativecommons. org/licenses/by/4.0/.

(c) The Author(s) 2021 